\title{
Addition of immunosuppression to supportive care does not STOP-IgAN
}

New data from the STOP-IgAN trial suggest that immunosuppressive treatment might not be beneficial in high-risk patients with IgA nephropathy (IgAN) who are receiving supportive care. This finding is in contrast to the results of previous studies that reported a potential benefit of immunosuppression in this population.

In the STOP-IgAN trial, Jürgen Floege and colleagues investigated whether the addition of immunosuppressive therapy (either glucocorticoid monotherapy or a regimen that included prednisolone, cyclophosphamide and azathioprine) to supportive care (renin-angiotensin system blockade with a bloodpressure target of $<125 / 75 \mathrm{mmHg}$, dietary counselling and advice to stop smoking and avoid nephrotoxins), was beneficial in patients with IgAN and persistent proteinuria $\geq 0.75 \mathrm{~g}$ per day, plus arterial hypertension and/or impaired renal function. The study consisted of a 6-month run-in phase, during which all participants received supportive care only, followed by a 3-year, open-label trial phase.

During the run-in phase, $34 \%$ of the 309 participants responded to supportive care with a reduction in proteinuria to $<0.75 \mathrm{~g}$ per day, and were, therefore, not eligible for inclusion in the trial phase. "For everyday clinical practice, this finding means that intensified, supportive therapy - that is, maximized antihypertensive and antiproteinuric medication - should always be provided initially," comments Floege.

In the trial phase, the researchers randomly assigned the remaining participants to receive either supportive care alone $(n=80)$ or supportive care plus immunosuppression $(n=82)$. At trial completion, $5 \%$ of patients in the supportive care group and $17 \%$ of those in the immunosuppression group had experienced a full clinical remission - defined as protein: creatinine ratio $<0.2$ and stable renal function with a decrease in estimated glomerular filtration rate (eGFR) of $<5 \mathrm{ml} / \mathrm{min} / 1.73 \mathrm{~m}^{2}$ from baseline $(P=0.01)$. However, no significant between-group difference was observed in decline in eGFR; $28 \%$ of patients who received supportive care had a decrease in eGFR $\geq 15 \mathrm{ml} / \mathrm{min} / 1.73 \mathrm{~m}^{2}$ compared with $26 \%$ of those who received immunosuppression $(P=0.75)$.

"There seems to be a benefit of immunosuppression for some patients with IgAN as indicated by the higher number of patients who achieved full clinical remission," comments Floege. "However, this benefit was not accompanied by any detectable effect of immunosuppression on loss of renal function, and whether it might translate into very long-term benefits is currently unknown." Importantly, the incidences of severe and nonsevere infections, malignant neoplasms, impaired glucose metabolism and weight gain $>5 \mathrm{~kg}$ during the first year of treatment were greater in the immunosuppression group than in the supportive care group. One patient who received immunosuppression died of sepsis.

"The STOP-IgAN study certainly raises a major question mark for immunosuppression," says Floege. "A key difference between the STOP-IgAN participants and patients in previously reported

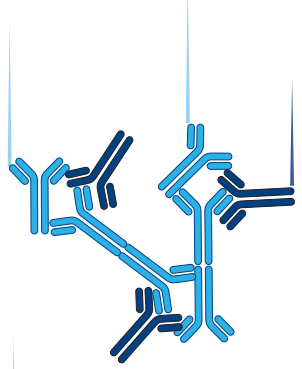

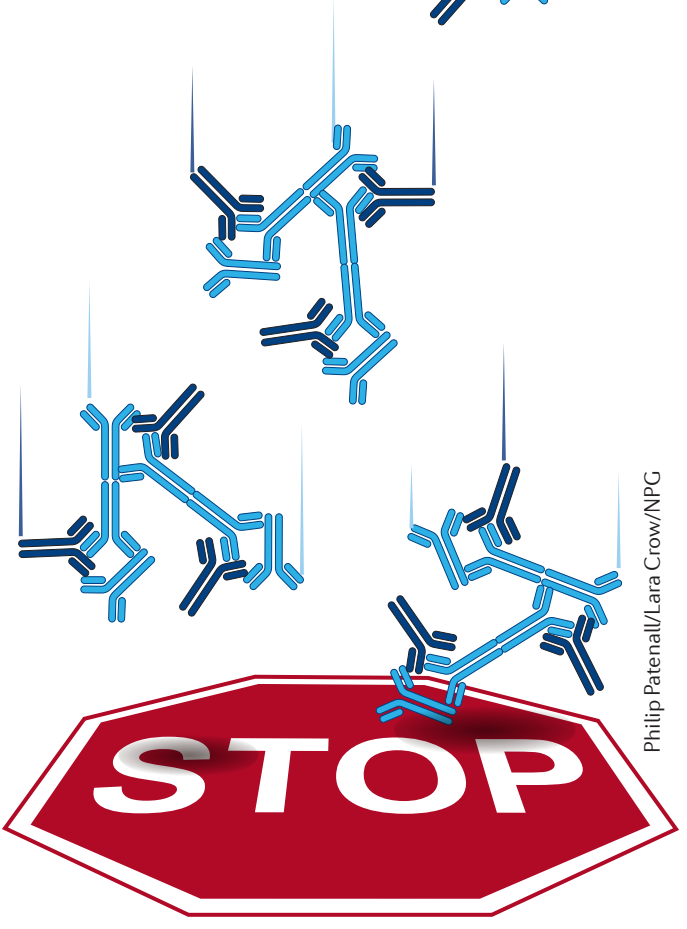

IgAN trials that showed a benefit of immunosuppressive therapy might be the very strict blood-pressure control that was achieved during the run-in phase and maintained during the trial period." Given the new findings, he suggests that if proteinuria persists at high levels ( $>3 \mathrm{~g}$ per day) and eGFR remains $>30 \mathrm{ml} / \mathrm{min} / 1.73 \mathrm{~m}^{2}$, corticosteroid monotherapy might still be consid-
The STOP-IgAN study certainly raises a major question mark for immunosuppression ered. In patients with lower degrees of proteinuria, the risks of systemic immunosuppression might outweigh any benefit and new therapies should be awaited.

Ellen F. Carney

ORIGINAL ARTICLE Rauen, T. et al. Intensive supportive care plus immunosuppression in IgA nephropathy. N. Engl.J.Med. 373, 2225-2236 (2015) 